PROCEEDINGS OF THE

AMERICAN MATHEMATICAL SOCIETY

Volume 129, Number 10, Pages 2833-2843

S 0002-9939(00)05882-2

Article electronically published on December 7, 2000

\title{
FINITE CONDUCTOR RINGS
}

\author{
SARAH GLAZ \\ (Communicated by Wolmer V. Vasconcelos)
}

\begin{abstract}
We extend the definition of a finite conductor domain to rings with zero divisors, and develop a theory of these rings which allows us, among other things, to provide examples of non-coherent finite conductor domains, and to clarify the behavior of non-coherent polynomial rings, group rings and fixed rings over coherent rings.
\end{abstract}

\section{INTRODUCTION}

The finite conductor property of a domain $R$, that is the finite generation of the conductor ideals $(I: J)$ for principal ideals $I$ and $J$ of $R$, embodies both factoriality properties and finiteness conditions. Indeed, the class of domains where $(I: J)$ is itself principal is precisely that of Greatest Common Divisor (GCD) domains, while the requirement that $(I: J)$ be finitely generated is a necessary condition for the coherence of a domain. For that reason the finite conductor property makes frequent appearance in the literature in investigations involving factoriality and also in investigations concerned with finiteness, coherent-like conditions, of domains. Both factorial domains (GCD domains, UFDs, etc.) and the variety of coherentlike conditions have a long history of being investigated in their own right or with an emphasis on interconnections among them.

Our interest in the relation between the finite conductor property and coherence was aroused by a remark of Gabelli and Houston [GH] concerning a domain $R$ : "To our knowledge, there are no known examples which prove that these properties are distinct". GH] investigates a number of coherent-like conditions in pullback rings. The nature of the conditions necessary to ensure that pullback rings are coherent, quasi coherent (a property falling between coherence and finite conductor defined in [BAD]), or finite conductor domains does not yield examples differentiating between coherence and the other two properties [GH]. The same phenomenon, for $D+M$ constructions, occurs in $[\mathrm{DP}$. The original purpose of this work was to generate examples of non-coherent finite conductor domains, and Examples 4.4, 5.2, and 5.3 do just that. In the process we got involved in a more general investigation of the relation between the finite conductor property and coherence. In Section 2 we extend the definitions of finite conductor and quasi coherent domains to rings with zero divisors, explore several of their basic properties, and clarify the relations between these notions for rings of small weak dimension. In particular we show that

Received by the editors November 8, 1999 and, in revised form, February 18, 2000.

2000 Mathematics Subject Classification. Primary 13A99, 13B25, 13D05, 13 F15.

Key words and phrases. Finite conductor, coherence, quasi coherence, G-GCD rings. 
a finite conductor $\operatorname{ring} R$ of w. $\operatorname{dim} R=2$ is quasi coherent. In Section 3 we define G-GCD rings, a new class of finite conductor rings, which generalize GCD domains, G-GCD domains defined in [AA], and coherent regular rings. We explore the nature of these rings through the interplay between finiteness, flatness and projectivity of ideals, and end the section with an example of a total ring of quotients which is not a finite conductor ring. Section 4 explores the interplay between the (quasi) coherence of a ring $R$ and finite conductor properties of the polynomial ring $R[x]$. In particular we prove that if $R$ is an integrally closed coherent domain, then $R[x]$ is quasi coherent (Theorem 4.1); and if $R$ is a coherent regular ring, then $R[x]$ is actually a G-GCD ring (Theorem 4.3). In general it is difficult for a ring $R$ to ascend coherence to $R[x]$ (see [G5). As far as we know, Theorem 4.1 and Theorem 4.3 are the first results showing that for a large class of coherent rings $R$ with non-coherent polynomial ring, $R[x]$ retains some coherent-like properties. We also exhibit an example of a local domain $R$ of $\mathrm{w} \cdot \operatorname{dim} R=2$ which is finite conductor (in fact GCD) domain but not coherent. In Section 5 we exhibit conditions under which a fixed subring $R^{G}$ preserves the quasi coherence and finite conductor properties of the ring $R$. We provide an example of a non-coherent UFD fixed ring, and utilizing the results of Gilmer and Parker [G2, GP], we provide a sequence of non-coherent UFD group rings with strictly increasing Krull dimensions.

\section{Flatness}

Let $R$ be a ring, and let $Q(R)$ denote the total ring of quotients of $R$. For (fractionary) ideals $I$ and $J$ of $R,(I: J)$ denotes the conductor of $J$ into $I$. If $I=a R$ and $J=b R$ we write $(a: b)$ for $(I: J) . \mu(I)$ denotes the cardinality of a minimal set of generators of $I$. For a (fractionary) ideal $I$ of $R, I^{-1}=\{a \in Q(R) \mid a I \subset R\}$.

$R$ is a finite conductor ring if $a R \cap b R$ and $(0: c)$ are finitely generated ideals of $R$ for all elements $a, b$ and $c$ of $R$. $R$ is a quasi coherent ring if $a_{1} R \cap \cdots \cap a_{n} R$ and $(0: c)$ are finitely generated ideals of $R$ for any finite set of elements $c$ and $a_{1}, \ldots, a_{n}$ of $R$.

Proposition 2.1. Let $R$ be a ring. The following conditions are equivalent:

1. $R$ is a finite conductor ring.

2. Any (fractionary) ideal $I$ of $R$ with $\mu(I) \leq 2$ is finite presented.

3. $(a: b)$ is a finitely generated ideal of $R$ for all elements $a$ and $b$ of $Q(R)$.

If $R$ is a domain the above three properties are equivalent to:

4. $I^{-1}$ is finitely generated for any (fractionary) ideal $I$ with $\mu(I) \leq 2$.

In case $R$ is a domain the quasi coherence of $R$ is equivalent to the finite generation of $I^{-1}$ for any finite generated (fractionary) ideal $I$ of $R$.

Recall that a ring $R$ is a coherent ring if $(0: c)$ and $I \cap J$ are finitely generated for every $c \in R$ and any two finitely generated ideals $I$ and $J$ of $R$. Coherent rings are the classical examples of finite conductor (and quasi coherent) rings. UFDs and GCD domains are finite conductor domains (and since in both cases $a R \cap b R$ is a principal ideal for all $a$ and $b$ in $R$, these domains are also quasi coherent). So are Generalized Greatest Common Divisor (G-GCD) domains, defined in [AA]. A domain $R$ is a $G-G C D$ domain if the intersection of any two invertible ideals of $R$ is an invertible ideal of $R$. As this property extends to the intersection of finitely many invertible ideals such a domain is also quasi coherent. There are finite conductor domains $R$ for which not all $a R \cap b R$ are invertible ideals of $R$. One such Noetherian domain is $R=k\left[x^{2}, x^{3}, y, x y\right]$, where $k$ is a field and $x$ and $y$ are indeterminates 
over $k$. Then $m=\left(x^{2}, x^{3}, y, x y\right)$ is a non-invertible, in fact $m m^{-1}=m$, maximal ideal of $R$, and $x^{2} R \cap x^{3} R=x^{3} m$ is not invertible.

Faithfully flat ring extensions descend both the finite conductor and the quasi coherence properties. But if $A \subset B$ are rings and $B$ is merely flat over $A$, the extension does not need to descend the finite conductor property. To see this let $A=k\left[x, y x, y w, y^{2} w, y^{3} w, \ldots\right] \subset B=k[x, y]$, where $k$ is a field, $x$ and $y$ are indeterminates over $k$, and $w=y x+1$. Then $B$ is flat over $A$ [G4], but $A$ is not a finite conductor domain as $(y x: x)=\left(y x, y w, y^{2} w, y^{3} w, \ldots\right)$ is not a finitely generated ideal of $A$.

Let $\left\{R_{i}\right\}$ be a family of rings. Set $R=\prod R_{i}$ and let $I=\prod I_{i}$ and $J=\prod J_{i}$ be two ideals of $R$. Then $I \cap J=\prod\left(I_{i} \cap J_{i}\right)$. Similarly, for an element $c=\left(c_{i}\right)$ in $R,(0: C)=\prod\left(0: c_{i}\right)$. Thus $I \cap J$ is a finitely generated ideal of $R$ if and only if $\sup \left\{\mu\left(I_{i} \cap J_{i}\right)\right\}<\infty$; and $(0: c)$ is finitely generated if and only if $\sup \left\{\mu\left(\left(0: c_{i}\right)\right)\right\}<$ $\infty$. In particular if each $R_{i}$ is a finite conductor (respectively a quasi coherent) ring, then $R=\prod_{i=1}^{n} R_{i}$ is a finite conductor (respectively a quasi coherent) ring.

If $S$ is a multiplicatively closed subset of $R$, and $R$ is a finite conductor (respectively a quasi coherent) ring, then clearly $R_{S}$ is a finite conductor (respectively a quasi coherent) ring. In particular every localization of a finite conductor (respectively a quasi coherent) ring at a maximal ideal is a finite conductor (respectively a quasi coherent) ring. The converse holds for a ring $R$ with finitely many maximal ideals $m_{i}$. To see this, note that in this case the $\operatorname{ring} T=\prod R_{m i}$ is a finite conductor (respectively a quasi coherent) ring which is faithfully flat over $R$. But the converse does not hold in general. [MZ, Example 2.1] exhibits a domain which is locally factorial, but not a finite conductor domain.

We now turn our attention to rings of small weak dimension. Rings $R$ of w. $\operatorname{dim} R=0$ are precisely the von Neumann regular rings and as such coherent. Recall that a ring $R$ is called semihereditary if finitely generated ideals of $R$ are projective.

Proposition 2.2. Let $R$ be a ring of $\mathrm{w} \cdot \operatorname{dim} R=1$. The following conditions are equivalent:

1. $R$ is a semihereditary ring.

2. $R$ is a coherent ring.

3. $(0: c)$ is a finitely generated ideal of $R$ for every element $c$ of $R$.

In particular a domain $R$ of $\mathrm{w} . \operatorname{dim} R=1$ is a coherent ring.

The equivalence of 1 and 2 in Proposition 2.2 is well known, while 3 implies 1 follows from G5. Theorem 4.2.10, Corollary 4.2.6, and Corollary 4.2.19]. Thus for a ring of weak dimension 1 the finite conductor, the quasi coherence, and the coherence properties coincide. If the weak dimension of $R$ is two, this is not necessarily true.

Theorem 2.3. Let $R$ be a ring of $\mathrm{w} \cdot \operatorname{dim} R=2$. If $R$ is a finite conductor ring, then $R$ is a quasi coherent ring.

Proof. The proof follows from the following observations:

1. If $R$ is a finite conductor ring, then $I \cap J$ is a finitely generated ideal of $R$ for any finitely generated flat ideals $I$ and $J$ : Set $I=\left(a_{1}, \ldots, a_{n}\right)$ and $J=\left(b_{1}, \ldots, b_{m}\right)$ by [G5. Lemma 4.2.1], $I$ and $J$ are projective and hence free (principal) at every localization of $R$ by a prime ideal. Let $P$ be a prime ideal of $R$. Then $I R_{P}=a_{i 1} R_{P}$, $J R_{P}=b_{j 1} R_{P}$ for some $i=i_{1}$ and $j=j_{1}$. Then $I R_{P} \cap J R_{P}=a_{i 1} R_{P} \cap b_{j 1} R_{P}=$ 
$\left(a_{i 1} R \cap b_{j 1} R\right) R_{P} \subset\left(a_{i} R \cap b_{j} R, i=1, \ldots, n ; j=1, \ldots, m\right) R_{P} \subset(I \cap J) R_{P}$. Thus $I \cap J=\left(a_{i} R \cap b_{j} R, i=1, \ldots, n ; j=1, \ldots, m\right)$, and is therefore finitely generated.

2. If $R$ is a ring of w. $\operatorname{dim} R=2$ and for an element $c$ of $R,(0: c)$ is a finitely generated ideal, then $c R$ is a projective ideal of $R$ : Since w. $\operatorname{dim} R=2, \mathrm{w} \cdot \operatorname{dim}_{R} c R \leq$ 1 and thus $(0: c)$ is a finitely generated flat ideal of $R$. Let $P$ be a prime ideal of $R$. Then either $(0: c) R_{P}=0$ or $(0: c) R_{P} \neq 0$ and so it is a projective, and hence free, ideal of $R_{P}$. But then $c(0: c)=0$ implies that $c R_{P}=0$, therefore $(0: c) R_{P}=R_{P}$. We conclude that $(0: c)$ is a pure ideal of $R$, and so $c R \approx R /(0: c)$ is a flat ideal of $R$. Since $c R$ is finitely presented it is projective.

3. If $R$ is a ring of w. $\operatorname{dim} R=2$, then $I \cap I$ is a flat ideal of $R$ for any flat ideals $I$ and $J$ : Follows from the exact sequence $0 \rightarrow I \cap J \rightarrow I \oplus J \rightarrow(I, J) \rightarrow 0$, and the fact that any ideal of $R$ has weak dimension at most 1 .

4. If $R$ is a finite conductor ring of w. $\operatorname{dim} R=2$, any intersection of finitely many finitely generated flat ideals of $R$ is a finitely generated flat ideal of $R$ : The proof follows by induction on $n$, the number of finitely generated flat ideals intersected, with the case $n=2$ clear from 1 and 3 .

We remark that if $\mathrm{w}$. $\operatorname{dim} R=2$, the finite conductor, and hence quasi coherence, property does not necessarily imply coherence, even in case $R$ is a local domain. This is shown in Example 4.4.

\section{G-GCD RINGS}

Let $R$ be a ring, and let $Q(R)$ be the total ring of quotients of $R$. A fractionary ideal $I$ of $R$ is called invertible if $\Pi^{-1}=R$. It is well known that a fractionary ideal $I$ is invertible if and only if $I$ is a projective $R$ module containing a non-zero divisor. Motivated by the proof of Theorem 2.3 we define:

A ring $R$ is called a $G$-GCD ring if the following two conditions hold:

C1. Every principal ideal of $R$ is projective.

$\mathrm{C} 2$. The intersection of any two finitely generated flat ideals of $R$ is a finitely generated flat ideal of $R$.

Note that $\mathrm{C} 1$ is equivalent to: $(0: c)$ is a finitely generated ideal and $c R$ is a flat ideal for every element $c$ of $R$. In the presence of $\mathrm{C} 1$, an argument like the one employed in the proof of Theorem 2.3 shows that $\mathrm{C} 2$ becomes equivalent to: The intersection of any two principal (fractionary) ideals of $R$ is a finitely generated flat (fractionary) ideal of $R$. Also note that if $R$ is a domain the above definition coincides with the definition of a G-GCD domain. It is clear that G-GCD rings are quasi coherent rings.

Proposition 3.1. Let $R$ be a G-GCD ring. Then the following hold:

1. $R$ is a reduced ring and $R_{P}$ is a GCD domain for every prime ideal $P$ of $R$.

2. $R$ is integrally closed in its total ring of quotients.

3. Min $R$, the set of all minimal prime ideals of $R$, is compact in the induced Zariski topology.

4. $Q(R)$, the total ring of quotients of $R$, is a von Neumann regular ring.

Proof. 1. $R_{P}$ is a domain for any prime ideal $P$ of $R$ G5, Theorem 4.2.2], and hence $R$ is a reduced ring. Let $I$ and $J$ be two principal ideals of $R_{P}$. Then $I=a R_{P}$ and $J=b R_{P}$ for some $a$ and $b$ in $R$. Since $a R \cap b R$ is finitely generated and flat, so is $I \cap J$. Thus $I \cap J$ is principal. This implies that $R_{P}$ is a GCD domain. 
2. Let $c=a / b$ be an element of $Q(R)$ integral over $R$, and let $P$ be a prime ideal of $R$. Since $b$ is a non-zero divisor, $b \neq 0$ in $R_{P}$, and thus either $a=0$ in $R_{P}$ or $c$ is an element of the field of quotients of $R_{P}$ which is integral over $R_{P}$, and so an element of $R_{P}$. It follows that $(b: a) R_{P}=R_{P}$ for every prime $P$. Thus $c \in R$.

3. and 4. follow from [G5, Theorem 4.2.10].

G-GCD rings are well behaved with respect to faithfully flat extensions. To see this let $A \rightarrow B$ be a ring extension with $B$ faithfully flat over $A$ and assume that $B$ is a G-GCD ring. Since $B$ is a faithfully flat extension of $A, A$ is a finite conductor ring. If $I$ is an ideal of $A$ and $I B=I \otimes_{A} B$ is a flat ideal of $B$, since $B$ is faithfully flat over $A, I$ is a flat ideal of $A$. Thus $c A$ is flat and finitely presented, and therefore projective, for every $c$ in $A$; and if $I$ and $J$ are two finitely generated flat ideals of $A$, then $I B \cap J B$ being finitely generated and flat over $B$ implies that $I \cap J$ is finitely generated and flat over $A$. We conclude that $A$ is a G-GCD ring. The example given in Section 2 shows that extensions that are merely flat may not descend the G-GCD property.

At first glance it seems that we could define two stronger generalizations of a G-GCD domain by requiring that principal ideals of $R$ are projective and replacing $\mathrm{C} 2$ by either of the following:

$\mathrm{C} 2{ }^{\prime}$. The intersection of two finitely generated projective ideals of $R$ is a finitely generated projective ideal of $R$.

$\mathrm{C} 2{ }^{\prime \prime}$. The intersection of two invertible ideals of $R$ is an invertible ideal of $R$.

We will show that neither of these requirements generates a new class of rings. We first cite a theorem of Gruson [V2] which will be used several times in this paper:

Theorem $3.2(\mathrm{~V} 2])$. Let $R$ be a ring and let $S$ be an $R$ algebra of finite presentation. Let $M$ be a finitely generated $S$ module and let $T$ be a ring containing $R$. If $M \otimes_{R} T$ is a finitely presented $S \otimes_{R} T$ module, then the set $U$ of all points $P \in$ Spec $S$ for which $M_{P}$ is $R_{P \cap R}$ flat is open in Spec $S$ and $M$ is of finite presentation over $U$.

\section{Theorem 3.3.}

1. Let $R$ be a ring whose principal ideals are projective and let $I$ be a finitely generated flat ideal of $R$. Then $I$ is a projective ideal. In particular, $R$ is a G-GCD ring if and only if $\mathrm{C} 1$ and $\mathrm{C} 2^{\prime}$ are satisfied.

2. Let $R$ be a ring whose principal ideals are projective. If $a R \cap b R$ is finitely generated projective for any two non-zero divisors $a$ and $b$ of $R$, then $a R \cap b R$ is finitely generated projective for any two elements $a$ and $b$ of $R$. In particular, $R$ is a G-GCD ring if and only if $\mathrm{C} 1$ and $\mathrm{C} 2{ }^{\prime \prime}$ are satisfied.

Proof. 1. Since principal ideals of $R$ are projective, $Q(R)$, the total ring of quotients of $R$, is a von Neumann regular ring. Let $I$ be a finitely generated flat ideal of $R$. In the setting of Theorem 3.2 take $S=R, T=Q(R)$ and $M=I$. $T$ is coherent and thus $I T=I \otimes_{R} T$ is a finitely presented ideal of $T$. It follows that $I$ is finitely presented over Spec $R$ in the Zariski topology. We conclude that $I$ is finitely presented and therefore a projective ideal.

2. Let $a$ and $b$ be elements of $R$. Since principal ideals of $R$ are projective we can write $a=a^{\prime} e$ and $b=b^{\prime} f$, where $a^{\prime}$ and $b^{\prime}$ are non-zero divisors and $e$ and $f$ are idempotents [J]. We claim that $a R \cap b R=\operatorname{ef}\left(a^{\prime} R \cap b^{\prime} R\right)$. Clearly the right hand side is contained in the left hand side. For the converse let $x \in a R \cap b R$. A 
computation shows that $x=x e=x f=x e f \in e f\left(a^{\prime} R \cap b^{\prime} R\right)$. By the hypothesis $I=a^{\prime} R \cap b^{\prime} R$ is finitely generated and projective, and $I=e f I \oplus(1-e f) I$. Thus $a R \cap b R=e f I$ is finitely generated and projective.

As benefits a generalization of GCD domains, not all coherent rings are G-GCD rings (see the example following Proposition 2.1). Neither are all G-GCD rings coherent (Examples 4.4, 5.2, and 5.3), but there is an important class of coherent rings which are G-GCD rings. Recall that a ring $R$ is called regular if every finitely generated ideal of $R$ has finite projective dimension. This notion, which agrees with the classical definition of regularity in case the ring is Noetherian, has been extensively studied for coherent rings as well (see G5 for an extensive treatment and bibliography). Coherent rings of finite weak dimension are regular rings, though the converse does not necessarily hold. Using [V1, Theorem 3.17] we obtain:

Proposition 3.4. Let $R$ be a coherent regular ring. Then $R$ is a G-GCD ring.

For a ring $R$, its total ring of quotients $Q(R)$ is, in many ways, simpler than $R$ itself. In particular if $R$ is a, not necessarily coherent, G-GCD ring, then $Q(R)$ is a von Neumann regular ring, and thus coherent. Hence it seems interesting to note that a ring $R$ which is a total ring of quotients, that is, every element of $R$ is either a unit or a zero divisor, does not necessarily have to be a finite conductor ring. The construction of the following example originates in Quentel's paper Q1. Because of errors in this, otherwise excellent, paper (some of which were corrected in Q2 ), we refer the reader to the fully corrected version in [G5, Chapter 4, Section 2].

Example 3.5. Let $K$ be a countable, algebraically closed field, let $I$ be an arbitrary finite set, and let $N$ be the natural numbers. For two sets $A$ and $B$ denote by $A^{B}$ the set of all set maps from $B$ to $A$. Let $S=W(R) \subset K^{I \times N^{N}}$ be the algebra constructed in [G5, page 118]. $S$ satisfies the following properties [G5:

1. $S$ is a reduced ring.

2. $S=Q(S)$.

3. $\operatorname{Min} S$ is compact.

4. $S$ is not von Neumann regular.

We will show that a ring $S$ satisfying these four properties has an element $c$ in $S$ such that $(0: c)$ is not a finitely generated ideal of $S$. Since Min $S$ is compact but $Q(S)$ is not von Neumann regular there is an element $c$ in $S$ such that $c S$ is not a flat ideal of $S$ [G5, Theorem 4.2.10]. Assume that $(0: c)$ is a finitely generated ideal of $S$, and let $I=c S+(0: c)$. Since the ring is reduced $I=c S \oplus(0: c)$, and $I \nsubseteq P$ for any prime ideal $P$ of Min $S$ [G5. Lemma 3.3.4]. Since Min $S$ is compact and $I$ is finitely generated $I \nsubseteq \bigcup P$, as $P$ runs over all the minimal primes of $S$ M]. Thus $I$ contains a non-zero divisor. Since $S=Q(S), I=S$ and therefore $c S$ is a projective ideal. We conclude that $(0: c)$ is not a finitely generated ideal of $S$. (We remark in passing that since $R$ is reduced, but not a von Neumann regular ring, Krull $\operatorname{dim} R>0$.)

\section{Polynomial Rings}

It is well known that if $R$ is a UFD (respectively a GCD domain), then $R[x]$ is a UFD (respectively a GCD domain) (see [G1]). In [AA], the Andersons proved that if $R$ is a G-GCD domain, then so is $R[x]$. Regarding coherence the situation is more complicated. Recall that a coherent ring $R$ is called stably coherent if the polynomial rings $R\left[x_{1}, \ldots, x_{n}\right]$ are coherent for every $n$. It is known that von 
Neumann regular rings, semihereditary rings, hereditary rings, and coherent rings of global dimension two are stably coherent rings (see [G5. Chapter 7] for an extensive treatment and bibliography on this topic). Soublin [S] provided an example of a coherent ring $R$ (not a domain) of $\mathrm{w} \cdot \operatorname{dim} R=2$, for which $R[x]$ is not coherent. Alfonsi [A] refined Soublin's example to the case where $R$ is a domain. All the rings mentioned are coherent regular rings. As mentioned in the introduction, Theorem 4.1 and Theorem 4.3 below show that for a large class of coherent rings $R$, over which polynomial rings may not be coherent, the polynomial rings retain some coherent-like properties.

Theorem 4.1. Let $R$ be an integrally closed coherent domain. Then $R[x]$ is a quasi coherent domain.

Proof. Let $f_{1}, \ldots, f_{n}$ be elements of $R[x]$, and let $I=f_{1} R[x] \cap \cdots \cap f_{n} R[x]$.

Case 1. $I \cap R=J \neq 0$. Then a direct computation shows that $I=J R[x]$. Degree considerations force $f_{1}, \ldots, f_{n}$ to belong to $R$. It follows that $J$, and therefore $I$, is finitely generated.

Case 2. $I \cap R=0$. Let $K$ denote the field of quotients of $R$. In this case $I K[x] \neq K[x]$, thus $I K[x]=f K[x]$ for a polynomial $f \in I$. Denote by $c(f)$, the so called content of $f$, the ideal of $R$ generated by the coefficients of $f$. Let $d \in R$ be such that $d c(f)^{-1} \subset R$, and consider the divisorial ideal of $R[x], I_{1}=d f^{-1} I$. $I_{1} K[x]=K[x]$ and so $I_{1} \cap R=J_{1} \neq 0$. By Q3, Lemma 2], $I_{1}=J_{1} R[x]$. Since $I=d^{-1} f J_{1} R[x]$, to show that $I$ is finitely generated it suffices to show that $J_{1}$ is finitely generated. Clearly $J_{1}=I_{1} \cap R=\left\{r \in R \mid r f R \subset d f_{1} R[x] \cap \cdots \cap d f_{n} R[x]\right\}$.

Let $\operatorname{deg} f=m$ and $\operatorname{deg} f_{i}=n_{i}$, write $m-n_{i}=m_{i}$ and denote by $S_{k}$ the $R$ submodule of $R[x]$ consisting of all polynomials of degree less or equal to $k$. If, for an element $r$ in $R, r f=f_{i} g$ for some $g$ in $R[x]$, then $g$ necessarily belongs to $S_{m i}$. Thus $J_{1}=\left\{r \in R \mid r f R \subset d f_{1} S_{m 1} \cap \cdots \cap d f_{n} S_{m n}\right\}$. Now $d f_{i} S_{m i} \approx S_{m i}$ and is, therefore, a coherent submodule of the coherent (finitely generated free) $R$ module $S_{m}$. Thus $M=d f_{1} S_{m 1} \cap \cdots \cap d f_{n} S_{m n}$ is a coherent submodule of $S_{m} . N=f R$ is also a coherent submodule of $S_{m} . J_{1}=\left(M:_{R} N\right)=\left(0:_{R} N+M / M\right)$. To show that $\left(0:_{R} M+N / M\right)$ is a finitely generated ideal of $R$ it suffices to show that $M+N / M$ is a coherent $R$ module [G5, Corollary 4.1.7]. Consider the following exact sequences of $R$ modules:

$$
\begin{gathered}
0 \rightarrow N \cap M \rightarrow M \rightarrow M / M \cap N \rightarrow 0, \\
0 \rightarrow N \cap M \rightarrow N+M \rightarrow N+M / N \cap M \rightarrow 0, \\
0 \rightarrow M / N \cap M \rightarrow N+M / M \rightarrow N+M / N \cap M \rightarrow 0 .
\end{gathered}
$$

Since $R$ is a coherent ring and $M$ and $N$ are coherent submodules of a coherent $R$ module both $N \cap M$ and $N+M$ are coherent modules. Repeated use of G5. Theorem 2.5.1] yields the coherence of the $R$ module $N+M / M$. We conclude that $J_{1}$, and thus $I$, is indeed finitely generated.

It is not clear if the result of Theorem 4.1 can be extended to rings $R$ with zero divisors. As Querre's result Q3] seems to play a major role in our proof, a step toward answering this question, and a question of interest in its own right, will be to obtain an extension of Querre's result to rings with zero divisors. This seems for the moment intractable, nevertheless in case $R$ is a coherent regular ring we can bypass the difficulty, and ascend the G-GCD property to $R[x]$. 
Lemma 4.2. Let $R$ be a ring whose principal ideals are projective. Then $R[x]$ satisfies the same property. In addition, if $f \in R[x]$, then $(0: f)=e R[x]$ for an idempotent $e$ in $R$.

Proof. We first prove the following: Let $R$ be a reduced ring and let $f$ and $g$ be two elements of $R[x]$. Write $f=a_{n} x^{n}+\cdots+a_{0}$ and $g=b_{m} x^{m}+\cdots+b_{0}$. If $f g=0$, then $a_{i} b_{j}=0$ for all $i=1, \ldots, n$ and $j=1, \ldots, m$.

Proof. We first show by induction on $j$ that $a_{0} b_{j}=0$ for $j=1, \ldots, m$. Clearly $a_{0} b_{0}=0$. Assume that $a_{0} b_{0}=\cdots=a_{0} b_{k}=0$. As $f g=0$ the $k+1$ coefficient of $f g$ equals 0 , thus $a_{0}\left(\sum_{i+j=k+1} a_{i} b_{j}\right)=0$. It follows that $\left(a_{0} b_{k+1}\right)^{2}=0$. Since $R$ is a reduced ring $a_{0} b_{k+1}=0$. We now use induction on $i$ to finish the argument, carrying on the induction step by replacing $f$ with $\left[f-\left(a_{k} x^{k}+\cdots+a_{0}\right)\right] / x^{k+1}$.

Now, since principal ideals of $R$ are projective, $R$ is a reduced ring. Let $f=$ $a_{n} x^{n}+\cdots+a_{0}$ be an element of $R[x]$. By the above we have that $\left(0:_{R[x]} f\right)=J R[x]$, where $J=\bigcap_{i}\left(0: a_{i}\right)$. Since $a_{i} R$ is a projective ideal of $R,\left(0: a_{i}\right)$ is generated by an idempotent of $R$, say $e_{i}$. It follows that $J$ itself is generated by the idempotent $e=e_{1} \cdots e_{n}$. Thus $\left(0:_{R[x]} f\right)=e R[x]$, and $f R[x]$ is projective.

Theorem 4.3. Let $R$ be a coherent regular ring. Then $R[x]$ is a $G-G C D$ ring.

Proof. By Lemma [4.2, $R[x]$ is a ring whose principal ideals are projective. By Theorem 3.3 it suffices to show that if $f$ and $g$ are two non-zero-divisors in $R[x]$, then $I=f R[x] \cap g R[x]$ is an invertible ideal of $R[x]$. Since $I$ contains a non-zerodivisor, $f g$, it suffices to show that $I$ is a projective ideal of $R[x]$.

We first show that $I$ is a flat ideal of $R[x]$. Let $Q$ be a prime ideal of $R[x]$, and let $P=Q \cap R$. Then $R_{P}$ is a GCD domain, thus $R_{P}[x]$ is a GCD domain. $R[x]_{Q}=R_{P}[x]_{Q R_{P}[x]}$. Thus $I$ is locally flat and therefore flat.

Next we show that $I$ is finitely generated. Consider the exact sequence of $R[x]$ modules: $0 \rightarrow I \rightarrow f R[x] \oplus g R[x] \rightarrow(f, g) \rightarrow 0$. If $P$ is a prime ideal of $R$, since $R_{P}[x]$ is a GCD domain, $I_{P}$ is principal and non-zero, thus a free $R_{P}[x]$ module. Let $I_{P}=h R_{P}[x]$ for $h$ in $I$. Then $h=h^{\prime} e$, where $h^{\prime}$ is a non-zerodivisor in $R[x]$ and $e$ is an idempotent in $R$, where $(0: h)=(1-e) R[x][J]$. Let $F_{0}=f R[x] \oplus g R[x]$, and $F_{1}=h^{\prime} R[x]$. The map $F_{1} \rightarrow h R[x]$ (which takes $h^{\prime}$ to $h$ ) and the inclusion map $h R[x] \rightarrow I$ compose to yield the complex of $R[x]$ modules: $0 \rightarrow F_{1} \rightarrow F_{0} \rightarrow(f, g) \rightarrow 0$, with $F_{0}$ and $F_{1}$ finitely generated and free.

As $h R_{P}[x]=h^{\prime} R_{P}[x]$, localizing this complex at $P$ we obtain an exact sequence. Let $Q$ be a prime ideal of $R[x]$ with $Q \cap R=P$. Then localizing the complex at $Q$ we obtain an exact sequence. By [A, Proposition 4.2] there is a neighborhood of $Q$ in Spec $R[x]$, such that localizing the complex at any prime ideal in this neighborhood we obtain an exact sequence. We conclude that $(f, g)$ is finitely presented over Spec $R[x]$ in the Zariski topology, and therefore finitely presented. We conclude that $I$ is finitely generated.

Next we show that $I$ is finitely presented. Since $T=Q(R)$ is a von Neumann regular ring, $T[x]$ is a coherent (semihereditary) ring. In the setting of Theorem 3.2, let $S=R[x], T=Q(R)$ and $M=I$ to obtain that $I$ is a finitely presented ideal of $R[x]$.

Thus $I$ is a projective ideal of $R[x]$.

We now exhibit an example of a local non-coherent GCD domain $R$ of w. $\operatorname{dim} R=$ 2 . 
Example 4.4. We first consider Soublin's example $[\mathrm{S}]$. Let $S_{i}=Q[[t, u]]$ be countable many copies of the power series in two variables $t$ and $u$ over the rational numbers $Q$, and let $S=\prod S_{i}$. It is shown in $[\underline{S}$ that $S$ is a coherent ring of w. $\operatorname{dim} S=2$ and that the polynomial $\operatorname{ring} S[x]$ is not a coherent ring. Since $S$ is a coherent regular ring, $S[x]$ is a G-GCD ring, but $S[x]$ is neither a domain, nor a local ring and w. $\operatorname{dim} S[x]=3$. According to $\mathrm{A}$, Corollary 4.4], there is a localization $S_{P}$ of $S$, such that $S_{P}[x]$ is not a coherent ring. As a localization of a regular coherent ring, $S_{P}$ is a domain. Since $S_{P}[x]$ is not coherent w. $\operatorname{dim} S_{P}=2$. Thus w. $\operatorname{dim} S_{P}[x]=3$. To knock down the weak dimension by one and obtain a local ring we consider the ring $R=S_{P}(x)=S_{P}[x]_{P S_{P}[x]}$. Since $S_{P}[x]$ is not a coherent ring, $R$ is not a coherent ring by [G6 Theorem 1]. w. $\operatorname{dim} R=$ w. $\operatorname{dim} S_{P}=2$ by [G6, Theorem 2]. Clearly $R$ is a GCD domain.

\section{FIXED RINGS}

Let $R$ be a ring, let $G$ be a group of automorphisms of $R$, and denote by $R^{G}$ the fixed ring of $R$. $R^{G}=\{a \in R \mid g(a)=a$ for all $g \in G\}$. The conditions under which a coherent ring $R$ descends coherence to $R^{G}$ were explored in [G7]. A crucial restriction involves the existence of a module retraction map $\alpha: R \rightarrow R^{G}$, that is, $\alpha$ is an $R^{G}$ module homomorphism satisfying $\alpha(a)=a$ for all $a$ in $R^{G}$. If a module retraction map from $R$ to $R^{G}$ exists we say that $R^{G}$ is a module retract of $R$. Bergman $[\mathrm{B}]$ pointed out the existence of such a map in two cases:

1. $G$ is a finite group and $o(G)$, the order of $G$, is a unit in $R$.

2. $G$ is a locally finite group, that is, for every $a \in R$ the orbit of $a, G a$, has finite cardinality $n(a)$, and $n(a)$ is a unit in $R$ for every $a \in R$.

Along the same lines as [G7] we can show:

Proposition 5.1. Let $R$ be a finite conductor (respectively a quasi coherent) ring. Then $R^{G}$ is a finite conductor (respectively a quasi coherent) ring in the following cases:

1. $G$ is a locally finite group and $R$ is a flat $R^{G}$ module.

2. $R^{G}$ is a module retract of $R$ and $R$ is a flat $R^{G}$ module.

3. $R^{G}$ is a module retract of $R$ and $R$ is a finitely generated $R^{G}$ module.

The conditions exhibited in Proposition 5.1 under which the finite conductor, quasi coherence and coherence properties descend from $R$ to $R^{G}$ are shown to be a pretty tight fit by the multitude of examples provided in [G7] of coherent rings $R$ which do not descend coherence to $R^{G}$ because $R^{G}$ is not a finite conductor ring. Nevertheless there is an example where neither of the conditions of Proposition 5.1 hold but $R$ descends UFDness to $R^{G}$, without descending coherence. This is a variation on the famous example of Nagarajan [N].

Example 5.2. Example of a local non-coherent UFD of Krull dimension 2.

Let $F$ be the field $F=Z_{2}\left(\left\{a_{i}\right\},\left\{b_{i}\right\}\right)$, where $Z_{2}$ is the prime field of characteristic 2 , and $\left\{a_{i}\right\}$ and $\left\{b_{i}\right\}$ are infinitely many variables over $Z_{2}$. Let $S=F[x, y]_{(x, y)}$, where $x$ and $y$ are indeterminates over $F$. Set $p_{i}=a_{i} x+b_{i} y$, and define an automorphism $g$ of $S$ by $g(x)=x, g(y)=y, g\left(a_{i}\right)=a_{i}+y p_{i+1}, g\left(b_{i}\right)=b_{i}+x p_{i+1}$, for all $i$. Let $G=\langle g\rangle$, then $o(G)=2$, but 2, of course, is not a unit in $S$. Let $R_{0}=S^{G}$. (The original example of Nagarajan defines $S$ to be $F[[x, y]]$.) $R_{0}$ is a local Krull domain of Krull dimension 2. The proof of [N] that shows that $R_{0}$ is not Noetherian for the power series case translates verbatim to show that $R_{0}$ is 
not Noetherian in our case. The proof in [HL2, page 272] that shows that $R_{0}$ is strongly Laskerian in the power series case translates verbatim to show that $R_{0}$ is strongly Laskerian in our case. We made use of this fact to show that $R_{0}$ is not a coherent ring in the power series case [G7, page 2639], and the same proof applies to our case. Finally, it is shown in [HL1, page 111], that $R_{0}$ is a UFD. (We remark that in the power series case it is not known whether $R_{0}$ is a UFD. What one can say in this case is that $R_{0}$ satisfies ACC for principal ideals [HL2, Corollary 3.6], therefore if $R_{0}$ is a GCD domain, then it is a UFD. It is not known if this ring is even a finite conductor domain.)

If $\operatorname{Krull} \operatorname{dim} R=n$, and $x$ is an indeterminate over $R$, then $n+1 \leq \operatorname{Krull} \operatorname{dim} R[x]$ $\leq 2 n+1$ [G1], thus $R_{0}\left[x_{1}\right] \subset R_{0}\left[x_{1}, x_{2}\right] \subset \cdots$, for $R_{0}$ the ring in Example 5.2 and $\left\{x_{i}\right\}$ infinitely many indeterminates over $R_{0}$, provides a chain of non-coherent UFDs of strictly increasing Krull dimensions. A more interesting example of the same phenomenon can be constructed by using group rings.

Let $G$ be an abelian group. $G$ is said to be cyclically Noetherian if $G$ satisfies the ascending chain condition for cyclic subgroups. Let $R$ be a ring, let $G$ be an abelian group and denote by $R G$ the group ring of $G$ over $R$. Gilmer and Parker GP] showed that for a domain $R$ and a torsion free abelian group $G, R G$ is a UFD if and only if $R$ is a UFD and $G$ is cyclically Noetherian. This characterization is used in [G2 to construct non-Noetherian (but coherent-by [G3, Theorem 1]) UFDs of arbitrary Krull dimensions. In Example 5.3 we utilize the idea behind Gilmer's construction to exhibit a collection of non-coherent UFD group rings with strictly increasing Krull dimensions.

Example 5.3. Let $p$ be a fixed rational prime, and let $Q^{(p)}$ be the additive group of rationals whose denominators are non-negative powers of $p$. Let $\sigma$ be a $p$-adic integer which is not rational, and let $\sigma_{n}$ be a sequence of rational integers with $\sigma_{n} \equiv \sigma\left(\bmod p^{n}\right)$ for all $n$. Choose independent elements $a, b$ in $Q^{(p)} \oplus Q^{(p)}$ and put $a_{n}=p^{-n}\left(a+\sigma_{n} b\right)$ for all $n$. Let $H$ be the group generated by $b$ and the sequence $\left\{a_{n}\right\}$. For every integer $m \geq 2$, let $H_{m}=H$ if $m=2$ and $H_{m}=H \times F_{m-2}$ if $m>2$, where $F_{m-2}$ is a free group of rank $m-2$. It is shown in G2, GP] that $H_{m}$ are torsion free cyclically Noetherian groups with rank $H=2$ and, thus, rank $H_{m}=m$ for $m>2$. Let $R_{0}$ be the fixed ring of Example 5.2. By the above discussion the rings $R_{m}=R_{0} H_{m}$ are UFDs. It is shown in G2 that for a group $G$ with $\operatorname{rank} G=t>0$, Krull $\operatorname{dim} R G \geq \operatorname{Krull} \operatorname{dim} R+1$. Thus Krull $\operatorname{dim} R_{2} \geq 3$. For $m>2, R_{m}=R_{2} F_{m-2}=R_{2}\left[x_{1}, x_{1}^{-1}, \ldots, x_{m-2}, x_{m-2}^{-1}\right]$ is integral over the polynomial ring in $m-2$ variables $A_{m-2}=R_{2}\left[x_{1}+x_{1}^{-1}, \ldots, x_{m-2}+x_{m-2}^{-1}\right.$ ] G3. Lemma 1]. Therefore Krull $\operatorname{dim} R_{2}+m-2 \leq \operatorname{Krull} \operatorname{dim} A_{m-2}=\operatorname{Krull} \operatorname{dim} R_{m}$, and so the Krull dimensions of $R_{m}$ are strictly increasing. It follows from G3, Theorem 1 ] that if for a ring $R$ and an abelian group $G, R G$ is a coherent ring, then so is $R$. We conclude that the rings $R_{m}$ are not coherent rings.

The author wishes to thank the referee for helpful comments.

\section{REFERENCES}

[A] B. Alfonsi, Grade non-Noetherian, Comm. in Algebra 9 (1981), 811-840. MR 82j:13012

[AA] D. D. Anderson and D. F. Anderson, Generalized GCD domains, Comment. Math. Univ. St. Pauli 28 (1980), 215-221. MR 81j:13017

[BAD] V. Barucci, D. F. Anderson and D. Dobbs, Coherent Mori domains and the principal ideal theorem, Comm. in Algebra 15 (1987), 1119-1156. MR 88c:13015 
[B] G. M. Bergman, Groups acting on hereditary rings, Proc. London Math. Soc. 23 (1971), 70-82. MR 45:293

[DP] D. Dobbs and I. Papick, When is $D+M$ coherent? Proc. AMS 56 (1976), 51-54. MR 53:13203

[GH] S. Gabelli and E. Houston, Coherent-like conditions in pullbacks, Mich. Math. J. 44 (1997), 99-123. MR 98d:13019

[G1] R. Gilmer, Multiplicative ideal theory, Queens Papers in Pure and Appl. Math. 12, 1968. MR 37:5198

[G2] R. Gilmer, A two dimensional non-Noetherian factorial ring, Proc. AMS 44 (1974), 25-30. MR 49:281

[GP] R. Gilmer and T. Parker, Divisibility properties in semigroup rings, Mich. Math. J. 21 (1974), 65-86. MR 49:7381

[G3] S. Glaz, On the weak dimension of coherent group rings, Comm. in Algebra 15 (1987), 1841-1858. MR 88g:16024

[G4] S. Glaz, Factoriality and finiteness properties of subalgebras over which $k\left[x_{1}, \ldots, x_{n}\right]$ is faithfully flat, Comm. in Algebra 16 (1988), 1791-1811. MR 89e:13008

[G5] S. Glaz, Commutative coherent rings, Springer-Verlag Lecture Notes in Math. 1371, 1989. MR 90f:13001

[G6] S. Glaz, On the coherence and weak dimensions of the rings $R\langle x\rangle$ and $R(x)$, Proc. AMS 106 (1989), 579-598. MR 89k:13018

[G7] S. Glaz, Fixed rings of coherent regular rings, Comm. in Algebra 20 (1992), 2635-2651. MR 93j: 13007

[HL1] W. Heinzer and D. Lantz, The Laskerian property in commutative rings, J. of Algebra 72 (1981), 101-114. MR 83h:13025

[HL2] W. Heinzer and D. Lantz, Commutative rings with ACC on n-generated ideals, J. of Algebra 80 (1983), 261-278. MR 84g:13030

[J] S. Jondrup, Groups acting on rings, J. London Math. Soc. 8 (1974), 483-486. MR 49:10679

[M] E. Matlis, The minimal spectrum of a reduced ring, Ill. J. of Math. 27 (1983), 353-391. MR 84h:13010

[MZ] J. Mott and M. Zafrullah, On Prufer v-multiplication domains, Manuscripta Math. 35 (1981), 1-26. MR 83d:13026

[N] K. Nagarajan, Groups acting on Noetherian rings, Nieuw Arch. voor Wisk. 16 (1968), 25-29. MR 37:5202

[Q1] Y. Quentel, Sur la compacité du spectre minimal d'un anneau, Bull. Soc. Math. France 99 (1971), 265-272. MR 44:6685

[Q2] Y. Quentel, Erratum, Sur la compacité du spectre minimal d'un anneau, Bull. Soc. Math. France 100 (1972), 461. MR 47:8512

[Q3] J. Querre, Idéaux divisoriels d'un anneau de polynomes, J. of Algebra 64 (1980), 270-284. MR 83b:13007

[S] J.-P. Soublin, Un anneau cohérent dont l'anneau de polynomes n'est pas cohérent, C. R. Acad. Sc. Paris, Ser. A 267 (1968), A241-A243. MR 38:1123

[V1] W. V. Vasconcelos, Divisor theory in module categories, North-Holland Math. Studies 14, 1974. MR 58:16637

[V2] W. Vasconcelos, The rings of dimension two, Lecture Notes in Pure and Appl. Math. 22, Marcel Dekker, 1976. MR 55:324

Department of Mathematics, University of Connecticut, Storrs, Connecticut 06269

E-mail address: glaz@uconnvm.uconn.edu 